\title{
Evaluation of Wayfinding Aids Interface in Virtual Environment
}

\author{
Anna $\mathrm{Wu}^{1}$, Wei Zhang ${ }^{1}$, Bo $\mathrm{Hu}^{1}$, and Xiaolong Zhang ${ }^{2}$ \\ ${ }^{1}$ Lab of Virtual Reality \& Human Interface Technology, \\ Department of Industrial Engineering, Tsinghua University, 100084, Beijing, China \\ wanclubs@gmail.com, zhangwei@tsinghua.edu.cn, \\ bo-hu05@mails.tsinghua.edu.cn \\ ${ }^{2}$ College of Information Sciences and Technology, \\ Pennsylvania State University, University Park, 16802, PA, USA \\ lzhang@ist.spu.edu
}

\begin{abstract}
It is difficult for a navigator to find a way to a given target location in an unfamiliar environment. Often, wayfinding guidance such as an overview map is provided to assist the navigator. However, overview maps can only show survey knowledge at one particular scale, and cannot provide other kinds of spaital knowledge (e.g. procedure knowledge) or survey knowledge at different scales. In this study, we compared effectiveness, efficiency and satisfaction of three wayfinding aids, View-in-View Map (VVM), Animation Guide (AG) and Human-System Collaboration (HSC) in support of navigation in virtual reality. Our experiment results show that while an overview still outperforms AG and HSC, AG serves better for most people with ordinary spatial ability and people with superior spatial ability tends to perform better using HSC.
\end{abstract}

Keywords: Wayfinding, Virtual environments, Interactive techniques, Spatial cognition.

\section{Introduction}

Research on wayfinding in virtual environment is numerous and with highly diversified purposes. When a navigation destination is known, tools often focus on how to get a navigator to the destination quickly and accurately. For example, some tools, like logarithmic movement ${ }^{[1]}$, let systems execute viewpoint movement after the destination is specified. While these tools are efficient in movement, they deprive the navigator's opportunities to actively explore virtual environments.

Exploration is critical to some navigation. In situations, such as training spatial knowledge of places that are rare, remote or dangerous ${ }^{[2]}$, as well as geographical information visualization (GIS) and scientific data visualization ${ }^{[3][4]}$, a navigator often needs to explore the space during wayfinding to understand spatial relationship among objects in virtual environments. Such exploration can help constructing of a comprehensive cognitive map about the space, which is important to other spatial activities, such as choosing an alternative route when the planned is unavailable. 
This paper presents an experimental study to compare three wayfinding aids to support exploration-oriented navigation with a known destination. The paper first reviews relevant research in Section 2, and proceeds to the brief introduction of wayfinding aids of our interest in Section 3. Then, Section 4 describes the experimental study and presents the results in Section 5. After discussion in Section 6, we conclude the paper with future work.

\section{Related Work}

Most designs to support virtual environment navigation are based on human navigation behaviors in the real world. Three levels of spatial knowledge in navigation have been identified ${ }^{[5]}[6]$ : landmark, procedure, and survey knowledge. It has been found that spatial knowledge in virtual world is similar to that in real world [7] [8] [9] [10].

Massive research has been done to support wayfinding. Different wayfinding aids have been proposed for different activities. A tool that is effective and efficient in one situation may not be helpful in another. There is a need to understand what kinds of navigation activities that different wayfinding aids may support. Here, we classify these aids based on where a navigator wants to go and how to reach there. Thus, we can identify four kinds of navigation activities: reaching a known destination by exploration, reaching a known destination with a system tool, reaching an unknown destination with exploration, and reaching an unknown destination with a system tool. The last case is not meaningful, because without knowing the exact location of destination, systems tools would not work.

Often a navigator wants to get a known place quickly and does not need selfexploration. In such situations, system tools, like teleportation, can simply take the navigator from one place to another, which are preferable when efficiency is a concern. Nevertheless, the navigator is very passive in obtaining knowledge about the space between the origin and destination. However, in many situations, to have route knowledge and survey knowledge is indispensable.

If the destination is unknown and the navigator needs to explore the space to find the target, navigation aids usually focus on providing visual information about the environments. Visual information could be the overview of environments or significant landmarks ${ }^{[10]}$. An overview can be in $2 \mathrm{D}$ or $3 \mathrm{D}$ maps and both have been tested to be useful in assisting wayfinding activities in virtual world ${ }^{[11][12]}$. Map-based aids can also be used to show the user's real-time position. A You-Are-Here map ${ }^{[13]}$ dynamically updates position and orientation which help to keep the users' spatial awareness. If a separated overview is not preferred when screen estate is limited, zooming techniques can be applied ${ }^{[14]}$. Google earth is an application of using zooming to provide users with different levels of bird-views. However, it still requires people to establish a connection between 2D satellite images with 3D real architectures and such transition is impossible for users to replicate.

Sometimes, even the system knows the target location, it is still necessary for a navigator to get the destination in person. For example, in spatial knowledge training, firefighters and military soldiers need to transfer their spatial knowledge into the real world and thus exploration becomes important to building a cognitive map about the space so that trainees can deal with unexpected events, such as changing to a new 
route. Moreover, large-scale collaboration asks ${ }^{[15][16]}$ each user to explore the space within her own action domain in order to work with partners. Such exploration is often not naive search, because destination is usually known.

\section{The Considered Wayfinding Aids}

Because exploration-based navigation with a known target still possesses values, our research focuses on evaluating three tools that support such navigation activities. In this section, we introduce these tools briefly.

\subsection{Human-System Collaboration (HSC)}

Our HSC tool provides movement direction guidance to the navigator. Direction information is calculated automatically by the system based on the location of the target and the user. The system only presents direction information when necessary, such as at decision-making points like intersections. By following 'simple interaction' design criteria to limit the caused visual obstruction and extended application of query-based designs ${ }^{[17][18]}$, our design is aimed at creating a collaborative relationship between human users and system tools.

Fig. 1 shows the interface design of the HSC aids. Direction information is presented at the window label. By guiding the user's movement direction, such directional information may play an important role in helping the user mentally building a route.

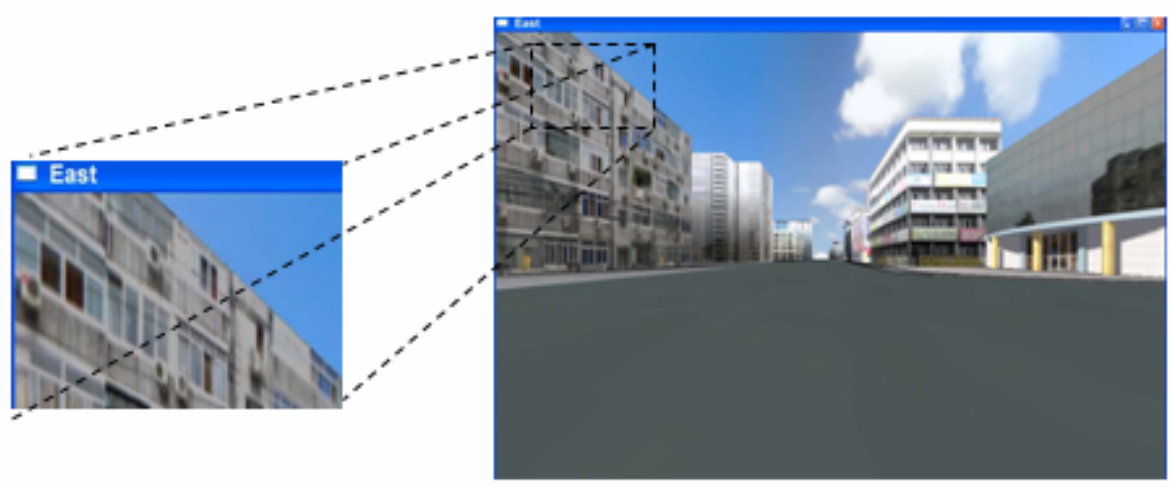

Fig. 1. HSC aid interface (Perspective lines added to show direction information, which tells the user that the target is on the east at that moment)

\subsection{Animation Guide (AG)}

An AG tool provides the user a dynamic view of a region through animation. Unlike an overview tool, which is static and is fixed at a particular scale, the AG tool shows how the user's starting point and the destination are connected with a series of views at different scales. Inspired by the space-scale animation approach ${ }^{[16]}$, our design of the AG tool first lifts the user's viewpoint from the starting point and then lowers it 


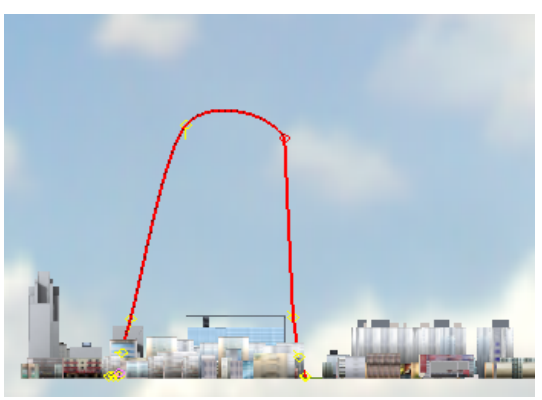

(a) Front view of animation guide trajectory

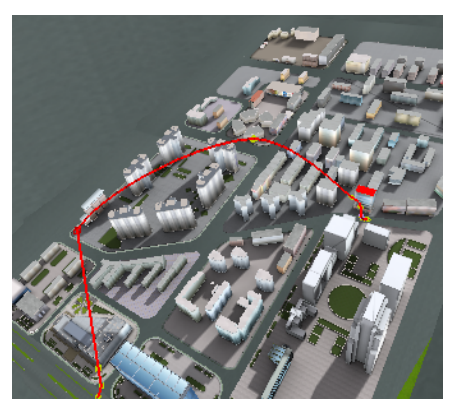

(b) $3 \mathrm{D}$ view of AG trajectory

Fig. 2. AG aid interface

on the way towards the target (Fig. 2). This AG tool not only allows the user to acquire landmark knowledge, also provides survey knowledge at different scales.

\subsection{View-in-View Map (VVM)}

A VVM tool shows the target location and a user's current location, as seen in Fig 3. In the VVM, the target is represented as a dot, and the user as a triangle. This VVM tool can be regarded as an overview map without detailed information, like buildings and streets. Such information is omitted because we focus on the guidance of the relative direction to the target, rather than survey knowledge.

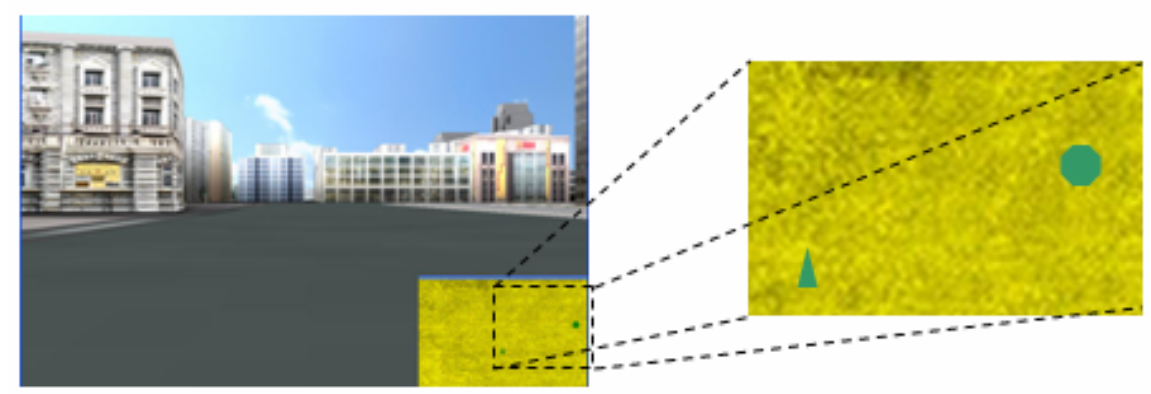

Fig. 3. VVM aid interface (Perspective lines added to show relative direction to the target)

\section{Experimental Study}

Our experimental study compared the effectiveness, efficiency and satisfaction of the above three tools in support of wayfinding.

\subsection{Experiment Design and Hypotheses}

The experiment had four treatments: the HSC tool, the AG tool, the VVM tool, and an environment without any wayfinding aid where only self-navigation ( $\mathrm{SN}$ ) is allowed to serve as a baseline condition. 
Wayfinding task used in the experiment had three difficulty levels: easy, medium, and difficult. The difficulty of the task was measured by the minimum traveling distance and the number of turns. By 'minimum', we mean the least necessary travel distance along street to get the target. For example, Fig. 4 shows a wayfinding task, which starts from the triangle and ends at the dot. The line illustrates the shortest path, and the length of this path is the minimum travel distance to complete this task. Only one turn is involved in this task. The minimum travel distances of tasks at three levels are $200 \mathrm{~m}, 400 \mathrm{~m}$, and $800 \mathrm{~m}$, respectively. The minimum turns are 0,1 , and 2 , respectively.

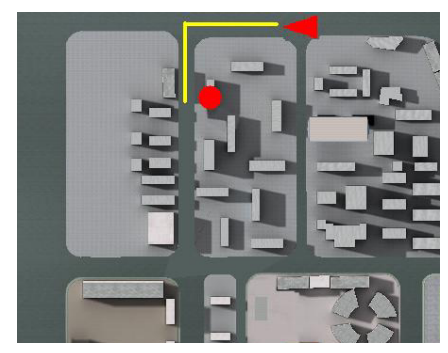

Fig. 4. Wayfinding tasks difficulty

The hypotheses of this research are:

- Wayfinding aids can improve subjects' performance in navigation, in terms of task completion time and error rate;

- The effectiveness, efficiency and satisfaction of different wayfinding aids vary because the different kinds of spatial knowledge they provide;

- The preference of these aids may depend on a subject's spatial ability.

\subsection{Subjects}

Twenty four (12 male and 12 female) college students participated in the study. Their ages were between 21 and 27, and the average was 23 .

\subsection{Apparatus}

The experiment was performed on a workstation (Pentium $4 \mathrm{CPU} 2.8 \mathrm{GHz}, 2.0 \mathrm{~GB}$ RAM). The machine had a NVIDIA Quadro FX1100 graphs card and a 19" ViewSonic Professional Series P95f monitor with a 1024 X 768 resolution. Subjects interacted with the virtual environment through a 3-button mouse and a standard keyboard. Keys needed in the experiment were labeled by tapes with action names.

\subsection{Test Scenes}

Four high fidelity different virtual city models were created. Each was used to test an aid interface or the non-aids situation, Self-Navigation (SN). The size of each model was $1500 \times 1000 \mathrm{~m}^{2}$. All models shared the common street layout and most of the 
background buildings. The only difference of four models was the target building type: one with three restaurants, one with three student association buildings, one with three banks and one with three bookstores. The target buildings in one city model did not appear in the others so that subjects would not meet the same target building in different models.

\subsection{Procedure}

Subjects were required to fill a background survey with detailed instructions upon arrival and then did two standard spatial ability tests, paperfolding test ${ }^{[19]}$ and Purdue Visualization of Rotations Test (ROT) ${ }^{[20]}$. We allowed as much time as possible for subjects in the training practice so that they could get familiar with the virtual environment and also were able to act freely using each interface. Subjects entered to perform formal tasks until two pretests were successively passed. For each task in the formal experiment, subjects were asked to find a named target building in the virtual city by using one of the interfaces. Each subject had 5 minutes maximum to perform a task and were asked to stop if they cannot find the target when time is up. Each subject took all the twelve tasks and success rate and performance time for each task were recorded. The order of all four treatments was counter-balanced to reduce the carry-over effect. After the test, subjects were asked to fill in a satisfaction questionnaire to evaluate their perception of different wayfinding aids.

\section{Results}

Fig. 5 presents aggregate task completion performance across different interfaces in terms of success rate (SR) and performance time (PT). For every task, a failure is recorded when the subject could not find the target in a maximal time of 5 minutes and 5 minutes is recorded as performance time for this task in our analysis.

Chi-Square test shows the SR of non-aids situation (SN) is approximated to $50 \%$ $\left(\chi_{1,24}^{2}=2.667, p=0.102\right)$. Chi-Square test found SRs of the three aids interfaces

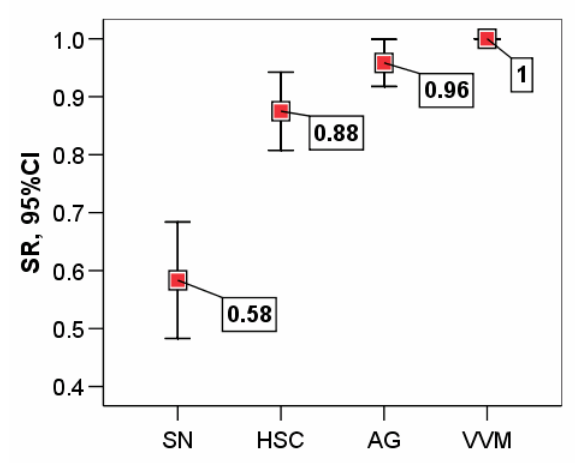

Fig. 5. Task Success Rate comparison 
had better subject performance than SN (HSC: $88 \%, \chi_{1,24}^{2}=54.000, p<0.001$; AG: $96 \%, \chi_{1,24}^{2}=80.667, \mathrm{p}=0.001$; and VVM: $\left.100 \%\right)$. Cochran's $\mathrm{Q}$ tests shows significant difference among the three aids interfaces $\left(Q_{2,72}=16.17, p<0.001\right)$. However, McNemar Test shows no statistical differences of SR between AG and VVM.

A two-way within-subject ANOVA testing performance time (PT) suggests significant main effect of aids interface type $\left(F_{2,193}=52.412, p<0.001\right)$ and task difficulty levels $\left(F_{2,19}=53.524, p<0.001\right)$ as shown in Fig. 6. A significant interaction effect between aids interface type and task difficulty is found as well $\left(F_{4,193}=14.256, p<0.001\right)$.

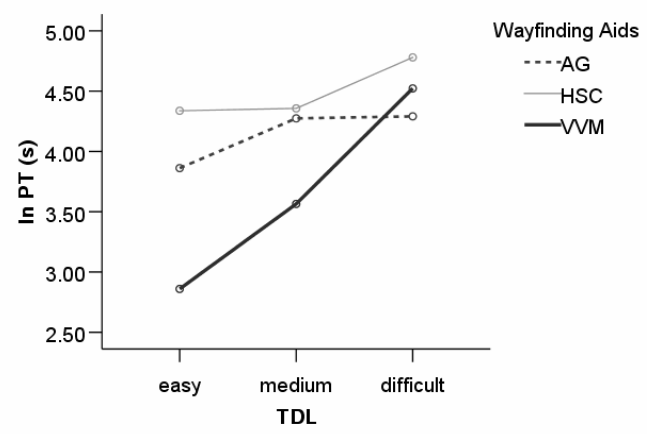

Fig. 6. Task completion comparison: Mean Performance Time in searching target (in logarithm) for different Task Difficulty Levels (TDL)

Fig. 7 illustrates the overall preference of the four interfaces. Frieman's test and Wilcoxon sign rank test showed significant overall satisfaction difference among the three interfaces $\left(\chi_{2}^{2}=35.03, p<0.001\right.$; for HSC and AG: $\left.Z=-2.711, p=0.017\right)$.

In addition to these three primary measurements, other effects were also observed. Pearson tests show that performance time has a negative correlation with score in spatial ability tests $(r=-0.53, p=0.008)$. Among the three aids interfaces, a strong correlation between subjects' performance using HSC aids interface and spatial ability exists $(r=-0.80, p<0.001)$.

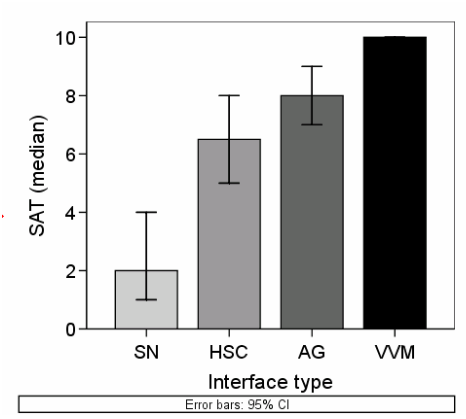

Fig. 7. Satisfaction ratings comparison 
Fig. 8 shows the distribution of different spatial ability subjects' performance time using AG and HSC. Most subjects with normal spatial ability spent significant less time finding the target with the AG tool than with the HSC tool $(58.3 \%$ of all 24 subjects), though subjects with low scores in spatial test still had to spend much time in both aids ( $25 \%$ of all 24 subjects). For those who had superior spatial ability watching animation was a time burden; they could search more efficiently by using the HSC aid interface (16.7\% of all 24 subjects).

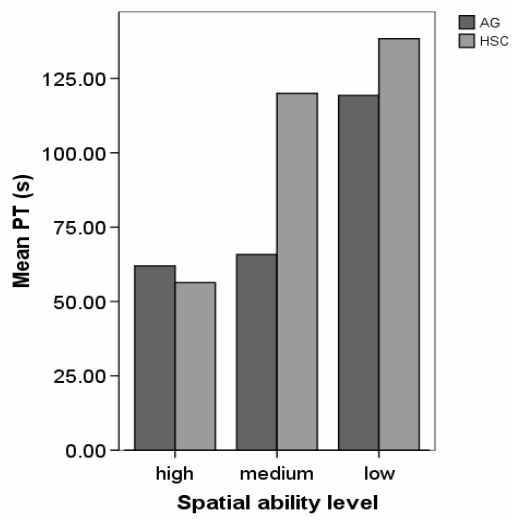

Fig. 8. Performance comparison between HSC and AG grouped by spatial ability

\section{Discussion}

Experiment results seem to indicate that wayfinding aids can greatly help users in exploration-based wayfinding in a virtual city. For the self-navigation condition, though 5 minutes is enough to navigate the whole city once without repeated route, subjects wandered aimlessly in the virtual city and could not relocate the buildings they have just visited. Without any wayfinding aid, the average success rate of finding the target is only approximate $50 \%$, where possibility is almost like get head after throwing a coin. As Witmer et. al ${ }^{[7]}$ has pointed out that spatial knowledge was developed more difficult in VE than real world.

Although different comments were addressed for the three aids, they all gained success rate above $85 \%$. By providing additional information about the relationship between the navigator and target position, wayfinding aids help users to form their cognitive map and thus facilitate their decision making and executing process in wayfinding. As shown in Fig. 6, the performance time with the VVM tool was almost linear to the necessary travel distance which may indicate that subjects seldom wasted time in extra wandering. Animation provides guidance for users to know where the target is and how to get there before searching by themselves, but users still possess risk to forget what they have seen in the animation and such risk made subjects nervous in later searching. Using the HSC tool, users need spatial ability to translate exo-centric direction information into ego-centric movement direction. Most subjects 
found it difficult to use the HSC tool. This might be due to the mental burden to do the translation.

\section{Conclusion and Future Work}

This paper presented a study to evaluate three wayfinding aids in support of exploration-based navigation in virtual environments. The results of our subject study are encouraging. We found that the three aids are effective and usable. We also found that these tools may benefit people with different spatial capabilities in different ways. The View-in-View Map aid is favorable in general; the Human-System-Collaboration tool suits people with high spatial ability better; and the Animation Guided aid is a beneficial alternative to people with normal or low spatial capabilities.

Future research efforts should be extended into two directions. First, we like to deepen our study by looking at wayfinding processes, such as how these tools may affect the assessment of wayfinding activities and how people translate exo-centric wayfinding guidance into ego-centric movement action. Second, we are interested in studying the individual preferences of wayfinding aids, especially in spatial capability. This paper has shown that wayfinding aids could have different impacts on people with different spatial capabilities, but it is still unclear why this happened.

\section{References}

1. Mackinlay, J.D., Card, S.K., Robertson, G.G.: Rapid Controlled Movement through a Virtual 3D Workspace. Computer Graphics 24(4), 171-176 (1990)

2. Bliss, J.P.: The Effectiveness of Virtual Reality for Administering Spatial Navigation Traning to Firefighters. Presence: Teleoperators and Virtual Environments 6, 73-86 (1997)

3. Bartram, L.: Perceptual and Interpretative Properties of Motion for Information Visualization. In: The 1997 Workshop on New Paradigms in Information Visualization and Manipulation, Las Vegas, NV, USA (1997)

4. Bjork, S.: Hierarchical Flip Zooming: Enabling Parallel Exploration of Hierarchical Visualizations. In: Proceedings of the Workshop on Advanced Visual Interfaces, Palermo, Italy (2000)

5. Downs, R.M., Stea, D.: Image and Environment: Cognitive Mapping and Spatial Behavior. Aldine Publishing, Chicago (1973)

6. Siegel, A.W., Herman, J.F: The Development of Spatial Representations of Large-scale Environments. In: Reese, H.W. (ed.) Advances in Child Development and Behavior, vol. 10, pp. 9-55. Academic Publisher, New York (1975)

7. Witmer, B.G., Bailey, J.H., Knerr, B.W., Parsons, K.: Virtual spaces and real world places: transfer of route knowledge. International Journal of Human-Computer Studies 45(4), 413-428 (1996)

8. Ruddle, R.A., Payne, S.J., Jones, D.M.: Navigating Buildings in Desk-Top Virtual Environments: Experimental Investigations Using Extended Navigational Experience. Journal of Experimental Psychology 3(2), 143-159 (1997)

9. Goerger, S.R., Darken, R.P., Boyd, M.A., Gagnon, T.A.: Spatial Knowledge Acquisition from Maps and Virtual Environments in Complex Architectural Spaces. In: Proceedings of the 16th Applied Behavioral Sciences, Colorado Springs, CO, USA (1998) 
10. May, M., Peruch, P., Savoyant, A.: Navigating in a Virtual Environment With MapAcquired Knowledge: Encoding and Alignment Effects. Ecological Psychology 7(1), 2136 (1995)

11. Darken, R.P., Sibert, J.L.: A Toolset for Navigation in Virtual Environments. In: Proceedings of ACM User Interface Software \& Technology, New York, NY, USA (1993)

12. Chittaro, L., Venkataraman, S.: Navigation Aids for Multi-floor Virtual Buildings: a Comparative Evaluation of Two Approaches. In: the ACM Symposium on Virtual Reality Software and Technology, Limassol, Cyprus (2006)

13. Levinew, M., Marchon, I., Hanley, G.: The Placement and Mispalcement of you-are-here Maps. Environment and Behavior 16(2), 139-157 (1984)

14. Buering, T., Gerken, J., Reiterer, H.: Usability of Overview Supported Zooming on Small Screens with Regard to Individual Differences in Spatial. In: the working conference on Advanced visual interfaces, Venezia, Italy (2006)

15. Zhang, X., Furnas, G.W.: mCVEs:Using Cross-Scale Collaboration to Support User Interaction with Multiscale Structures. Presence: Teleoperators and Virtual Environments 14(1), 31-46 (2005)

16. Zhang, X.: Space-Scale Animation: Enhancing Cross-Scale Understanding of Multiscale Structures in Multiple Views. In: Proceedings of Coordinated and Multiple Views in Exploratory Visualization, London, UK (2005)

17. Ballegooij, A. v., Eliens, A.: Navigation by Query in Virtual Worlds. In: Proceedings of the sixth international conference on 3D Web technology, Paderbon, Germany (2001)

18. Chittaro, L., Burigat, S.: 3D Location-pointing as a Navigation Aid in Virtual Environments. In: Proceedings of AVI 2004: 7th International Conference on Advanced Visual Interfaces, Gallipoli, Italy (2004)

19. Ekstrom, R.B., French, J.W., Prince, L.A.: Kit of Reference Tests for Cognitive Factors. Educational Testing Services Princeton, NJ (1963)

20. Bodner, G.M., Guay, R.B.: The Purdue Visualization of Rotation (ROT) Test. The Chemical Educator 2(4), 1-17 (1997) 